\title{
Alternative splicing in 5'-untranslational region of STK-15 gene, encoding centrosome associated kinase, in breast cancer cell lines
}

\author{
Sei One Shin', Kwan Ho Lee², Jung Hye Kim³, \\ Suk-Hwan Baek ${ }^{3}$, Jong-Wook Park ${ }^{4}$, \\ Edward W Gabrielson ${ }^{5}$ and Taeg Kyu Kwon ${ }^{4,6}$ \\ ${ }^{1}$ Department of Therapeutic Radiology and Oncology, \\ College of Medicine, Yeungnam University, Taegu 705-717, Korea \\ ${ }^{2}$ Department of Internal Medicine, College of Medicine, Yeungnam \\ University, Taegu 705-717, Korea \\ ${ }^{3}$ Department of Biochemistry and Molecular Biology, \\ College of Medicine, Yeungnam University, Taegu 705-717, Korea \\ ${ }^{4}$ Department of Immunology, School of Medicine, \\ Keimyung University, Taegu 700-712, Korea \\ ${ }^{5}$ Department of Pathology, Johns Hopkins University School of \\ Medicine, Baltimore, MD 21224, USA \\ ${ }^{6}$ Corresponding author: Tel, +82-53-250-7846; \\ Fax, +82-53-255-1398; E-mail, kwontk@dsmc.or.kr
}

Accepted 14 November 2000

Abbreviations: STK15, serine/threonine kinase 15; RT-PCR, reverse transcription-polymerase chain reaction; bp, base pair

\begin{abstract}
Centrosomes maintain genomic stability by establishing the bipolar spindles during cell division and execute accurate segregation of chromosomes during mitosis. In this study, we have demonstrated that there are three forms of STK-15 gene in breast cancer cell lines. Alternative splice positions are located in 5'-untranslated region of STK15 gene. The results of in vitro translation experiments revealed that the alternative splicing in the 5'-untranslated region of STK15 had no effect on protein translation. The differential expression patterns of these alternatively spliced STK15 in breast cell lines and primary tumors therefore suggest that STK15 gene transcription may be differentially regulated or stabilized in these cells.
\end{abstract}

Keywords: STK15, centrosome, breast cancer, alternative splicing, serine/threonine kinase, cell cycle

\section{Introduction}

Clear segregation of chromosomes during mitosis is an important regulatory event in the progression of cell cycle. A bipolar spindle composed of two centrosomes and a dynamic array of mircotubule assemblies and disassemblies carries out the chromosome segregation (Elledge, 1998; Hardwick, 1998). The functions of centrosome and spindle may be regulated by phosphorylation, since phosphorylation of centrosomal proteins in Drosophila and vertebrates were found to influence microtuble nucleation and dynamics at the centrosomes (Kellogg et al., 1995; Taagepera et al., 1995). Two related kinases, Ipl1 and aurora, were identified in budding yeast and flies respectively and mutational inactivation of these kinases cause missegregation of chromosome and disturbance of centrosome separation (Francisco et al., 1994; Glover et al., 1995).

STK15 (also known as BTAK and aurora 2) encodes a centrosome associated kinase and was identified in chromosome $20 q 13$, a region amplified in a number of human breast cancer cell lines (Sen et al., 1997; Bischoff et al., 1998). Expression of the STK15 protein and its kinase activity are regulated during cell cycle and both are at a highest levels during the $\mathrm{G}_{2} / \mathrm{M}$ phase (Kimura et al., 1997; Yanai et al., 1997; Bischoff et al., 1998; Kimura et al., 1999). Recently, Zhou et al. (1998) reported that overexpression of STK15 induced centrosome amplification, chromosomal instability, transformation in mouse NIH 3T3 cells. Deregulation of the centrosomes was implicated in chromosomal abnormalities (Fukasawa et al., 1996). Therefore, the abnormal numbers of centrosomes were observed in a variety of human cancers (Lingle et al., 1998).

In this study, we have found the alternative splicing in the 5'-untranslated region of STK15 gene in breast cancer cell lines. The alternative splicing of transcripts in the 5'-untranslated region does not affect protein translation. Interestingly, STK15 transcripts exhibit differential alternative splicing patterns in breast cancer cell lines and primary tumors. These results suggest that the expression levels of STK15 transcripts may be differentially regulated or stabilized in cell lines and primary tumors.

\section{Materials and Methods}

Cell culture and patient samples

The human cell lines, MDA231, MDA468, MDA361, MDA436 and MCF7 were obtained from the ATCC 
(Rockville, MD). The culture medium used throughout these experiments was Dulbecco's modified Eagle's medium containing $10 \%$ FCS and $100 \mu \mathrm{g} / \mathrm{ml}$ gentamicin. For the study, informed consents were obtained from those patients with confirmed diagnoses of breast tumor at the Johns Hopkins Bayview Hospital.

\section{Reverse transcription-PCR (RT-PCR)}

Total RNA was extracted from breast cancer cell lines and primary tumor samples using the Ambion Totally RNA kit (Ambion Inc., Austin, TX) according to the manufacturer's specifications. Two micrograms of total RNA was reverse-transcribed (RT-Superscript, Gibco BRL, Gaithersburg, MD) with random hexamers to generate cDNA. Two microliters of each cDNA mixture was amplified using 1 unit Taq polymerase and the following oligonucleotide primers: 5'-CCTGTAGGATACTGCTTGTTACT-3' (upstream primer) and 5'-TATGGCAGCCCTGGCTCAAGGATT-3' (downstream primer). PCR was performed for 30 cycles under the conditions of annealing at $57^{\circ} \mathrm{C}(45 \mathrm{~s})$, extension at $72^{\circ} \mathrm{C}(1 \mathrm{~min})$, and denaturing at $94^{\circ} \mathrm{C}$ (45 s) using a Perkin-Elmer thermocycler. One-tenth of each reaction mixture was electrophoresed through a $1 \%$ agarose gel.

\section{DNA sequence analysis}

Amplified cDNA fragments were isolated from gel slices using a Qiagen gel extraction kit and subcloned into the TOPO vector. Sequencing was performed by the dideoxynucleotide chain termination procedure using a double stranded plasmid DNA with a Sequenase kit version 2.0 (Gibco BRL, Gaithersburg, MD).

\section{In vitro translation}

In vitro translation products of alternatively spliced STK15 were generated with a TNT-coupled reticulocyte lysate system (Promega, Madison, WI) in the presence of 40 $\mu \mathrm{Ci}\left[{ }^{35}\right.$ S]methionine $(1000 \mathrm{Ci} / \mathrm{mmol}$; Amersham, Arlington Heights, IL) following the manufacturers instructions. In vitro translated proteins were separated in a $10 \%$ SDSpolyacrylamide gel. The gel was fixed in $50 \%$ methanol/ $10 \%$ acetic acid, incubated in $1 \mathrm{M}$ sodium salicylate $(\mathrm{pH}$ 6.0) for $30 \mathrm{~min}$, and exposed to an X-ray film.

\section{RT- PCR and Southern blot analysis}

RT-PCT products were separated in a $1.5 \%$ agarose gel and transferred to a GeneScreen Plus membranes (Biotechnology Systems, Boston, MA). The membrane was incubated in Church buffer at $62^{\circ} \mathrm{C}$ with $\left[\alpha^{-32} \mathrm{P}\right] \mathrm{dCTP}$. labeled full size of STK15 cDNA. The probe was labeled using the random primer method.

A

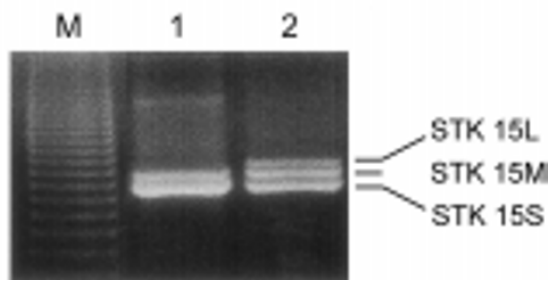

B

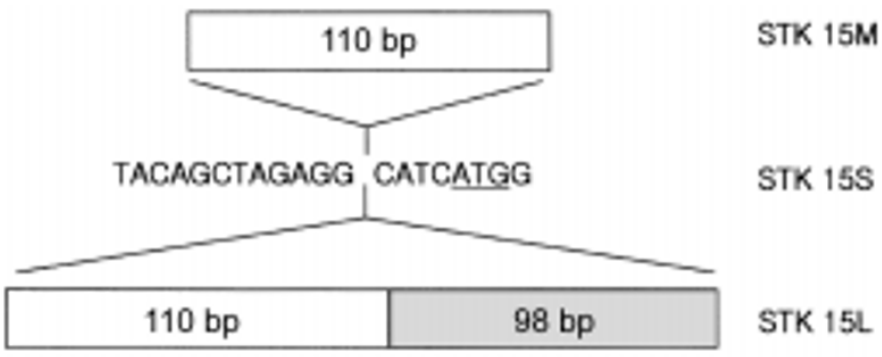

C

GTCTCACTCCATTGCCCAGGCCAGAGTGOGGGGATATTTGATAAGAAACTTCAGTG AAGGCCGGGCGCGGTGGCTCATGCCCGTAATCCCAGCATTTTCGGAGGOCGAGG....

D

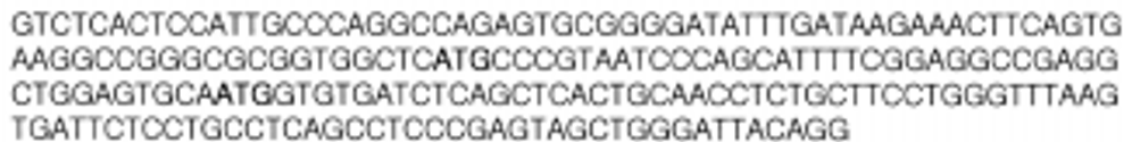

Figure 1. Alternative splicing generates three distinct mRNA transcripts. Primary tumor RNA samples and MDA 231 breast cancer RNA samples were incubated with reverse transcriptase and the resultant DNA was amplified by PCR as described under Materials and Methods. (A) Primary breast tumor (lane 1) and human MDA 231 (lane 2) RT-PCR yielded two and three sizes of cDNA (1295, 1305 and 1503 bp), respectively. (B) Schematic representation of the alternative splicing forms of STK15. (C) DNA sequence of 110 bp insert in 5'-untranslated region of the STK15M gene. (D) DNA sequence of 208 bp insert in 5'-untranslated region of the STK15L gene. The underline and bold letters indicate the translation start codon. 


\section{Results and Discussion}

\section{Alternative splicing of STK15 in breast cancer cell lines}

Recently, STK15 was found to encode a cell cycle regulating serine/threonine kinases that appears to be amplified and overexpressed in human breast cancers and colorectal cancers (Sen et al., 1997; Bischoff et al., 1998). Zhou et al. (1998) reported that overexpression of STK15 induced centrosome amplification and transformation in mouse NIH 3T3 cells. To determine whether chromosomal instability was linked to STK15 expression levels in breast cancer cells, we first cloned STK15 cDNA by RT-PCR using specific primers. These primers correspond to regions outside the STK15 coding region. RT-PCR using RNA of MDA231 human breast cancer cells yielded three distinct cDNA transcripts consisting of 1295, 1305 and $1503 \mathrm{bp}$. To further characterize the three MDA231 STK15 transcripts, the three RT-PCR products were subcloned into the TOPO vector and sequenced. Sequence analysis revealed that the shortest cDNA (referred to as STK15S) was identical to the STK15 mRNA (Genbank AF011468) reported in the literature (Zhou et al., 1998). The middle sized STK15 transcript (referred to as STK15M) and the largest STK15 (referred as STK15L) had a $110 \mathrm{bp}$ and $208 \mathrm{bp}$ insertion in 5'-untranslated region, respectively. These data suggest that the STK 15 gene was alternatively spliced in the 5'-untranslated region in the MDA 231 breast cancer cells.

\section{Alternatively spliced transcripts do not effect on protein translation}

Interestingly, the sequence differences among the three distinct STK15 transcripts were in the 5'-untranslated region which is the region critical for transcriptional and translation control. The alternative transcripts of STK15M and STK15L contain one or two additional translation start codons (ATG), respectively, in the 5'-untranslated region. To determine whether the $110 \mathrm{bp}$ and $208 \mathrm{bp}$ insertions in 5'-untranslated region had an effect on STK15 protein translation, each transcript subclone was transcribed and translated in vitro using the TNT-coupled reticulocyte system. The in vitro translated $\left[{ }^{35} \mathrm{~S}\right]$

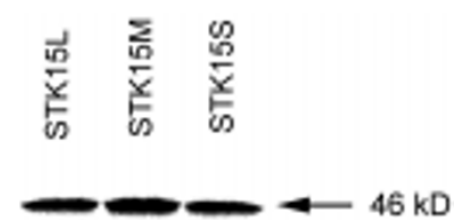

Figure 2. In vitro translation of the alternatively spliced STK15 gene. Alternatively spliced STK15-L, STK15-M and STK15-S were in vitro translated using the TNT-coupled reticulocyte system. The translation products were separated in a $10 \%$ SDS-polyacrylamide gel. The gel was fixed and exposed to X-ray film for 1 day.

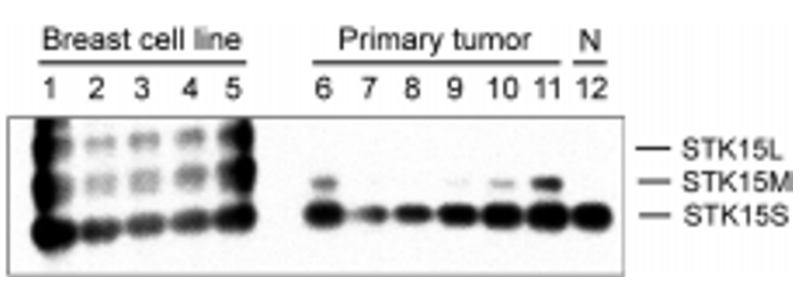

Figure 3. Alternatively spliced transcript patterns in breast cancer cell lines and primary tumors. Total RNA was isolated from 5 human breast cancer cell lines (lane 1, MCF7; lane 2, MDA231; lane 3, MDA361; lane 4, MDA468; lane 5, MDA436), 6 primary tumor samples (lane 6-11) and normal cell $(\mathrm{N})$. RT-PCR analysis was performed and the PCR products were hybridized to full length of CDNA probe.

methionine-labeled STK15 proteins were then analyzed by SDS-PAGE. As shown in Figure 2, alternatively spliced STK15 transcripts yielded the same sized protein and showed no difference in translational efficiency. These results suggest that the differences in the STK15 transcripts do not affect the protein code, although the transcripts maybe are generated by differential promoter usage and thus possibly differentially expressed in human tissues or cell type.

\section{Alternatively spliced transcript patterns in breast cancer cell lines and primary tumors}

In order to get an overview of the pattern of expression of the STK15 mRNA transcript, we analyzed different breast cancer cell lines, primary tumors and normal cells using RT-PCR. Single stranded cDNAs were synthesized from total RNA from various breast cancer cell lines, primary tumors and normal cells. The RT-PCR experiment used an upstream primer corresponding to position 215-237 of the STK15 cDNA sequence and a downstream primer corresponding to position 448-470 of the cDNA sequence. Results showed differential expression of STK15 alternatively spliced transcripts among the tested samples. To confirm the specificity of the PCR products, the PCR products were separated in an agarose gel and transferred by Southern blot to a membrane that was then hybridized with the cDNA probes. As shown in Figure 3, breast cancer cell lines had at least three alternatively spliced transcripts, while primary tumors contained two transcript forms. Interestingly, these results suggested that the STK15 gene exhibits alternative splicing patterns in cell lines and primary tumors that are distinctive.

In conclusion, our data indicate the existence of at least three STK15 transcript forms. Their expression level may be differentially regulated or stabilized in different cell lines and primary tumors.

\section{Acknowledgements}

This research was supported by the Yeungnam University Medical Center Research grants in 1999. 


\section{References}

Bischoff, J. R., Anderson, L., Zhu, Y., Mossie, K., Ng, L., Souza, B., Schryver, B., Flanagan, P., Clairvoyant, F., Ginther, C., Chan, C. S., Novotny, M., Slamon, D. J. and Plowman, G. D. (1998) A homologue of Drosophila aurora kinase is oncogenic and amplified in human colorectal cancers. EMBO J. 17: 3052-3065

Elledge, S. J. (1998) Mitotic arrest: Mad2 prevents sleepy from waking up the APC. Science 279: 999-1000

Francisco, L., Wang, W. and Chan, C. S. (1994) Type 1 protein phosphatase acts in opposition to IpL1 protein kinase in regulating yeast chromosome segregation. Mol. Cell Biol. 14: $4731-4740$

Fukasawa, K., Choi, T., Kuriyama, R., Rulong, S. and Vande Woude, G. F. (1996) Abnormal centrosome amplification in the absence of p53. Science 271: 1744-1747

Glover, D. M., Leibowitz, M. H., McLean, D. A. and Parry, H. (1995) Mutations in aurora prevent centrosome separation leading to the formation of monopolar spindles. Cell 81: 95105

Hardwick, K. G. (1998) The spindle checkpoint. Trends Genet. 14: $1-4$

Kellogg, D. R., Oegema, K., Raff, J., Schneider, K. and Alberts, B. M. (1995) CP60: a microtubule-associated protein that is localized to the centrosome in a cell cycle-specific manner. Mol. Biol. Cell 6: 1673-1684
Kimura, M., Kotani, S., Hattori, T., Sumi,N., Yoshioka, T., Todokoro, K.and Okano, Y. (1997) Cell cycle-dependent expression and spindle pole localization of a novel human protein kinase, Aik, related to Aurora of Drosophila and yeast Ipl1. J. Biol. Chem. 272: 13766-13771

Kimura, M., Matsuda, Y., Yoshioka, T. and Okano, Y. (1999) Cell cycle-dependent expression and centrosome localization of a third human aurora/lpl1-related protein kinase, AlK3. J. Biol. Chem. 274: 7334-7340

Lingle, W. L., Lutz, W. H., Ingle, J. N., Maihle, N. J. and Salisbury, J. L. (1998) Centrosome hypertrophy in human breast tumors: implications for genomic stability and cell polarity. Proc. Natl. Acad. Sci. U. S. A .95: 2950-2955

Sen, S., Zhou, H. and White, R. A. (1997) A putative serine/ threonine kinase encoding gene BTAK on chromosome 20 q13 is amplified and overexpressed in human breast cancer cell lines. Oncogene 14: 2195-2200

Taagepera, S., Campbell, M. S. and Gorbsky, G. J. (1995) Cell-cycle-regulated localization of tyrosine and threonine phosphoepitopes at the kinetochores of mitotic chromosomes. Exp. Cell Res. 221: 249-260

Yanai, A., Arama,E., Kilfin, G. and Motro, B. (1997) ayk1, a novel mammalian gene related to Drosophila aurora centrosome separation kinase, is specifically expressed during meiosis. Oncogene 14: 2943-2950

Zhou, H., Kuang, J., Zhong, L., Kuo, W. L., Gray, J. W., Sahin, A., Brinkley, B. R. and Sen, S. (1998) Tumour amplified kinase STK15/BTAK induces centrosome amplification, aneuploidy and transformation. Nat. Genet. 20: 189-193 